Meta

Journal des traducteurs

Translators' Journal

\title{
La langue des assurances
}

\section{Jean-Paul de Grandpré}

Volume 12, numéro 4, décembre 1967

Aspects de la terminologie

URI : https://id.erudit.org/iderudit/002497ar

DOI : https://doi.org/10.7202/002497ar

Aller au sommaire du numéro

Éditeur(s)

Les Presses de l'Université de Montréal

ISSN

0026-0452 (imprimé)

1492-1421 (numérique)

Découvrir la revue

Citer cet article

de Grandpré, J.-P. (1967). La langue des assurances. Meta, 12(4), 133-136.

https://doi.org/10.7202/002497ar

Ce document est protégé par la loi sur le droit d'auteur. L'utilisation des services d'Érudit (y compris la reproduction) est assujettie à sa politique d'utilisation que vous pouvez consulter en ligne.

https://apropos.erudit.org/fr/usagers/politique-dutilisation/
Cet article est diffusé et préservé par Érudit.

Érudit est un consortium interuniversitaire sans but lucratif composé de l’Université de Montréal, l'Université Laval et l'Université du Québec à Montréal. Il a pour mission la promotion et la valorisation de la recherche. https://www.erudit.org/fr/ 


\section{LA LANGUE DES ASSURANCES}

Pour véhiculer une pensée cohérente dans une discipline donnée, il faut un répertoire de mots justes et de tournures idiomatiques. La langue des assurances, qui au Canada a été pendant longtemps soustraite à cette exigence fondamentale, illustre, si l'on peut dire, cette nécessité par l'absurde: les interférences qui s'y manifestent sous forme de calques sémantiques et de structure la rendent souvent inintelligible pour les usagers canadiens.

Ce défaut de communication serait encore plus grand entre francophones canadiens et européens. Qu'un agent québecois dise à un collègue français (ou belge, ou suisse): «Je vends de l'assurance-vie ordinaire et de l'assurance-vie industrielle », et ce dernier n'y verra que du feu. Que son homologue européen dise à son tour: "J'exerce en grande branche et en branche populaire», il trouvera la tournure recherchée et les mots utilisés passablement saugrenus !

Nous pourrions donner de nombreux exemples où le manque de « correspondance » entre le français et le franco-canadien est à peu près total. Mais ne serait-ce pas insister inutilement sur un état de choses déjà trop inquiétant pour ne pas être perçu par les intéressés ? Nous nous attacherons plutôt à en rechercher brièvement les causes.

Comme l'a fait remarquer M. Gérard Parizeau, «certaines assurances sont nées et se sont développées en pays britanniques surtout». Cet appel de termes nouveaux a donné naissance en ces milieux à un vocabulaire "hermétique, mais auquel l'usage a donné un sens assez précis dans l'ensemble $»^{1}$. Qu'aux États-Unis,

1. Gérard Parizeau, "Une requête au surintendant des assurances: pour un vocabulaire officiel des assurances", A ssurances, 33e année, no 4, p. 320-321. 
on ait par la suite pris la relève; qu'on ait modifié et complété les réalités et, dans un esprit souvent différent, qu'on ait créé le complément terminologique nécessaire pour rendre compte de la nouvelle situation, ne facilite pas les choses. Nos voisins eux-mêmes sentent maintenant le besoin de débroussailler ce maquis: l'American Risk ou l'Insurance Association a même institué à cette fin une commission d'étude.

Voilà pour la langue de départ. Pendant ce temps, les pays francophones façonnaient leur propre terminologie. Reflétant une psychologie et un contexte social différents, les termes utilisés diffèrent souvent des vocables anglais. En voici un bref exemple. Le commerce des assurances est considéré comme un service, mais seul le français en fait état au niveau du vocabulaire. En anglais, on « achète » (buy) une assurance; on en «vend » (sell); et souvent, on appelle l'agent insurance salesman. Notre langue, par souci sans doute de dématérialiser ce genre d'opération, s'exprime autrement: en français, on souscrit une assurance; on en fait souscrire; et celui qui exerce la profession d'agent s'appelle producteur. Refuser ces expressions et leurs très nombreux provignements, c'est s'exposer à commettre de fréquentes erreurs de démarche et à créer des confusions.

L'établissement d'une terminologie d'assurance en milieu canadien doit donc faire entrer en ligne de compte deux réalités différentes et deux esprits en bien des points divergents. Mais là ne s'arrête pas la difficulté que présente un travail de cette na:ure. En condition de fonctionnement normal, les assurances utilisent des termes provenant de nombreuses disciplines, notamment le commerce, la finance, l'administration, le droit ${ }^{2}$. Or, dans chacun de ces domaines, la langue accuse chez nous un état de dégradation avancée, inscrite de très longue date dans nos « mœurs » linguistiques. Ces expressions, quoique ne relevant pas directement d'un lexique des assurances, doivent quand même être préalablement redressées et y être intégrées si l'on veut procurer un instrument valable aux rédacteurs et traducteurs soucieux de s'exprimer intelligiblement.

La constatation de ces différents registres d'achoppement permet, croyonsnous, d'établir les critères d'élaboration d'une saine terminologie des assurances. Il convient, selon nous, de poser la communication sous l'angle des vocables, bien sûr, mais aussi en fonction d'une démarche existante, caractérisée par un affaiblissement des mécanismes psychologiques, résultant de servitudes de symétrie fautives et acquises de longue date. De toute évidence, il faut plus que plaquer de simples étiquettes.

Nous avons, pour notre part, tenté d'illustrer cette méthode dans un champ restreint de la terminologie envisagée, celui de l'assurance sur la vie ${ }^{3}$. Sous l'angle matériel, disons qu'il nous a été impossible d'organiser un comité qui nous eût permis de faire appel aux connaissances d'autres traducteurs de même discipline. Puisant à même un fichier dressé au cours de nos années de pratique, nous avons rédigé ce lexique, puis nous l'avons soumis à différents spécialistes de la langue et à des experts en droit et en technique des assurances. Ce travail, qui sera publié sous peu par l'Office de la langue française, n'est pas exhaustif. Il comprend

2. Cf. Emission Langue vivante, fiches linguistiques, "Termes juridiques", Montréal, RadioCanada.

3. Vocabulaire bilingue des assurances sur la vie (en préparation). 
cependant bon nombre des termes les plus courants dans l'industrie de l'assurance sur la vie. Voici, à titre indicatif, l'une des rubriques de ce vocabulaire:

Opinion du risque (Insurability) - Jugement que se forme l'assureur sur le risque à prendre en charge.

Voilà un cas où deux expressions, l'une française et l'autre anglaise, rendent compte d'une même réalité en l'envisageant sous un angle différent. Opinion du risque se retrouve chez les bons auteurs: "L'assuré doit ... déclarer à l'assureur les circonstances lui permettant de se faire une opinion du risque » (Picard et Besson). «Il peut paraître étonnant de voir les assureurs s'attacher, pour se faire une opinion du risque, aux autres assurances » (idem). Les circonstances "de nature à changer l'objet du risque ou d'en diminuer l'opinion pour l'assureur $\gg$ (Code des assurances).

Toutefois, insurability ayant une extension plus grande que opinion $d u$ risque, il convient de procéder à des transpositions sémantiques si l'on veut rendre toutes les acceptions du vocable anglais. En voici quelques-unes, qui sont indiquées en caractères gras.

The occupation has particular importance in appraising insurability: $\mathrm{La}$ profession revêt une importance particulière pour l'appréciation du risque. To affect the insured's insurability: Aggraver le risque que présente l'assuré. Evidence of insurability satisfactory to the company: Une preuve établissant à la satisfaction de la compagnie que le candidat est assurable. Insurance against the possibility that one might lose his insurability: Une assurance contre la possibilité de devenir non assurable. If his insurability does not present too great an added risk: $\mathrm{Si}$ le risque qu'il présente ne comporte pas de circonstances trop aggravantes.

Assurable étant reçu pour désigner la qualité de celui qui peut être assuré, il semble bien que "assurabilité 》 pourrait désigner le fait d'être assurable. Force nous est de reconnaître pourtant que cette expression n'est pas encore entrée dans la langue officielle, même si elle se rencontre parfois en langue familière.

Voir aussi Déclaration de santé.

La lecture de cet article - le vocabulaire en question en comprend un peu plus de deux cents - permettra de relier la méthode utilisée à la situation décrite tout à l'heure. Aux erreurs ancrées par la force de l'habitude, nous avons opposé certains pouvoirs motivants: la définition d'abord de chacune des rubriques. En effet, ces définitions ne contribueront-elles pas à donner aux mots une dimension propre, à les concrétiser davantage, et de ce fait à en faciliter l'emploi ?

La mise en parallèle des expressions françaises et anglaises répond également à un souci d'explicitation: en milieu canadien-français, l'usager étant très souvent bilingue, le rapprochement des deux démarches fait ressortir la logique propre à notre langue et aide ainsi à faire valoir le bien-fondé du vocable français. Sous ce rapport, l'indication des formes fautives a également son utilité. Elle permet de juger de l'importance prise par les calques. Cette constatation devrait normalement provoquer l'effort nécessaire pour conjurer l'effet destructeur que ces fautes grossières exercent sur notre langue.

Cet effort sera grandement facilité si traducteurs et rédacteurs veulent bien se documenter. La normalisation intégrale d'un pareil secteur de la langue exige 
plus qu'un simple lexique: il faut le complément de lectures nombreuses et attentives ${ }^{4}$.

Il existe en France une maison se spécialisant dans la publication de textes d'assurances, la Librairie de l'Argus, dont on peut se procurer le catalogue en écrivant au 2, rue de Châteaudun, Paris, $\mathrm{IX}^{\mathrm{e}}$. On trouvera dans cette brochure, qui comprend plus de deux cents titres, des ouvrages touchant toutes les branches d'assurance et les domaines connexes: droit, comptabilité, fiscalité, enseignement, etc.

\section{BIBLIOGRAPHIE}

Annuaire des sociétés d'assurances, Paris, Éditions de l'Argus, 2100 p.

Assurances, revue trimestrielle consacrée à l'étude théorique et pratique de l'assurance au Canada, Montréal.

Baglin, P.-R., Manuel pratique du producteur d'assurance, Paris, Librairie générale de droit et de jurisprudence, 1960, $465 \mathrm{p}$.

Deschamps, Jacques, l'Agent général d'assurances, Paris, Librairie technique, $1958,197 \mathrm{p}$.

Faudue, Maurice, les Assurances, Paris, Presses Universitaires de France, "Que sais-je ? », $\mathrm{n}^{\circ} 76,1958,126 \mathrm{p}$.

Godart, Justin et André Perraud-Charmantier, Code des assurances, $3^{e}$ éd., Paris, Éditions techniques S.A., 1947, 685 p.

La Correspondance avec l'assureur, Paris, Centre de documentation et d'information de l'assurance, $47 \mathrm{p}$.

L'Argus, journal international des assurances, Paris.

Législation générale concernant les sociétés d'assurances en France, Paris, Éditions de l'Argus, $390 \mathrm{p}$.

Les Assurances, cours conforme au programme d'assurance des écoles supérieures de commerce, Paris, Centre de documentation et d'information de l'assurance, $55 \mathrm{p}$.

Parizeau, Gérard, Traité d'assurance contre l'incendie au Canada, Montréal, Beauchemin, 1961, $465 \mathrm{p}$.

PiCARD, Maurice et André Besson, les Assurances terrestres en droit français, 2 vol., vol. I: le Contrat d'assurances, 756 p.; vol. II: les Entreprises d'assurances, 390 p., Paris, Librairie générale de droit et de jurisprudence, 1965.

Villars, Christian, le Manuel de l'inspecteur et de l'agent, Paris, Éditions de l'Argus, 1964, 279 p.

JEAN-PAUL DE GRANDPRÉ

4. Nous publions sur cetie page une courte bibliographie qui pourrait servir utilement à cette fin. 